\title{
SYNTHESIS AND TRANSMUTATION OF STABLE AND RADIOACTIVE ISOTOPES IN BIOLOGICAL SYSTEMS
}

\author{
Alla A. Kornilova \\ Lomonosov Moscow State University, http://www.msu.ru \\ 1/2, Leninskie gory, Moscow 119991, Russian Federation \\ prfnart@mail.ru
}

\section{Vladimir I. Vysotskii}

Shevchenko Kiev National University, http:/ /www.univ.kiev.ua

64/13, str. Vladimirskaya, Kyiv 01601, Ukraine

vivysotskii@gmail.com

Abstract. The work considers the background, theoretical assumptions and results of experiments on nuclear transmutation of stable and radioactive isotopes in growing biological objects. It is shown that such fundamental transformations are based on nuclear-physical processes, the course of which is stimulated by the catalytic influence of non-stationary spatial inhomogeneities in the volume of growing living objects. This process can be used both for the accelerated utilization of stable and radioactive nuclei, and for the production of rare isotopes. The conditions for optimizing the deactivation process are investigated. It was found that in the most optimal case the rate of activity decrease in aqueous solutions of radionuclides is accelerated by $35 . .200$ times in relation to the natural decay. The physical and biological reasons of these processes are considered.

Keywords: isotopes, microbiological systems, low energy nuclear reactions, deactivation of radionuclides

PACS: 25.60.Pj; 25.70.-z; 28.41.Kw; 28.52.-s; 28.60.+s; 82.90.+j; 87.17.-d; 87.17.Ee; 87.80.-y; 89.60.-k

Bibliography -23 references

Received 13.06.2017

RENSIT, 2017, 9(1):52-64

DOI: $10.17725 /$ rensit.2017.09.052

\section{Contents}

1. INTRODUCTION (52)

2. Biological aspects of the process of TRANSMUTATION OF ELEMENTS (53)

3. EXPERIMENTS ON SYNTHESIS OF STABLE IZOTOPES IN MICROBIOLOGICAL SYSTEMS (54)

4. EXPERIMENTS ON TRANSMUTATION AND DETECTION OF RADIOACTIVE IZOTOPES IN MICROBIOLOGICAL SYSTEMS (56)

4.1. EXPERIMENTS ON UTILIZATION OF THE REACTOR IZOTOPE ${ }^{140} \mathrm{BA}_{\mathrm{A}}$ (58)

4.2. EXPERIMENTS ON UTILIZATION OF THE LONG-LIVED REACTOR ${ }^{137} \mathrm{Cs}$ IZOTOPE IN GROWING ASSOCIATIONS OF MICROBIOLOGICAL STRUCTURES (60)

5. Conclusion (62)

REFERENCES (63)

\section{INTRODUCTION}

The hypothesis of the possibility of nuclear transmutation of chemical elements and their isotopes in biological systems is one of the most mysterious in the history of science, it has often been discussed in recent decades and has its own history and mythology, its own supporters and critics. This problem arose in the "pre-nuclear period" and the initial stages of its emergence are closely connected with alchemy. A brief background of this phenomenon is presented in $[1,2]$.

Among the many predecessors, a series of works by L. Kervran [3-6] occupies a special place in the chronology of studies on the transmutation of chemical elements and isotopes in biological objects. In fact, he was the first scientist of the "nuclear age," who conducted a systematic study of the processes of transmutation of 
chemical elements in biological objects. In particular, Kervran investigated the potassium transmutation reaction in calcium ${ }^{39} \mathrm{~K}+{ }^{1} \mathrm{p} \rightarrow{ }^{40} \mathrm{Ca}$ in a biological system containing hydrogen. $\mathrm{He}$ also investigated many other reactions of isotope transmutation, among which one should especially note those that lead to the formation of vital micro- and macroelements $\mathrm{Ca}, \mathrm{K}, \mathrm{Mg}$, P. Many of the experiments of Kervran are very convincing if we consider them without analysis at the micro level.

At the same time, Kernran's scientific views were far enough from the requirements of nuclear physics. For example, it allowed the reversibility of the nuclear reaction ${ }^{39} \mathrm{~K}+{ }^{1} \mathrm{p} \leftrightarrow{ }^{40} \mathrm{Ca}$ and the possibility of the reactions of the $\mathrm{Cl}-\mathrm{O} \rightarrow \mathrm{F}$, $\mathrm{P}-\mathrm{Li} \rightarrow \mathrm{Mg}, \mathrm{Ca}-\mathrm{O} \rightarrow \mathrm{Mg}, \mathrm{Fe}-\mathrm{H} \rightarrow \mathrm{Mn}$ decay, which are incompatible with energy conservation laws, since for their realization requires a giant energy $Q \approx 5 \ldots 20 \mathrm{MeV}$, whose sources are absent in the biological environment! Kervran in his works treated such processes as a specific "biological transmutation," claiming that this is a very special process stimulated by an unknown enzyme. Such an interpretation (linking incomprehensible processes with yet another unknown enzyme) is quite common in biology, but it does little to understand and use these processes.

In our opinion, there is no reason to consider the process of isotope conversion in growing biological systems as a "biological transmutation" and separate it from the general physical concept of transmutation as a process of isotope conversion completely "controlled" by the laws of physics and stimulated by certain structural features of the biological system. Unfortunately, Kervran did not carry out in any of his experimental studies an analysis of the ratio of isotopes in the initial and final states. This is the basic error of Kervran's experiments, because nuclear physics is the science of isotopic (non-elemental!) transmutations - in other words, "nuclear physics is the chemistry of isotopes"!

\section{BIOLOGICAL ASPECTS OF THE PROCESS OF TRANSMUTATION OF ELEMENTS}

Specific biophysical aspects of the process of transmutation of isotopes in biological systems have been considered in detail in monographs $[7,8]$. It is possible to note only some of them. Experiments to study the transmutation of isotopes in growing biological systems should be conducted taking into account the specific of the problem and the following requirements:

- The synthesized isotope must be included in the number of micro- and macroelements vital to the biosystem of or their biochemical analogs, and the medium in which growth occurs should not contain these elements, but must contain those isotopes from which these elements can be formed without irreversible additional energy generation.

- In order for experiments to be conducted in a short time, the growth rate of the biological object under study must be large, and the isotope formed must be adapted to this object and must accumulate in an amount sufficient for reliable registration.

- The living object must be sufficiently resistant to the action of the products of vital activity in order to avoid the phenomenon of self-intoxication.

- When working with radioactive isotopes, the living object must be sufficiently resistant to the action of strong radiation fields formed by the utilized isotopes.

- It is desirable (and for stable isotopes mandatory) the possibility of analyzing the isotope composition of a biological object before and after the transmutation process.

- The requirements related to the environment and to the problems of humanism (we are talking about living objects) must be met.

The best objects for such experiments are microbiological cultures, the characteristics 
of which meet the above requirements to the maximum extent. As a rule, "pure" microbiological cultures can effectively grow only under certain environmental conditions (acidity, temperature, the presence of toxins, radiation background, etc.), and if these conditions are violated, growth is sharply inhibited or becomes impossible. The typical dose that conventional microbiological cultures can withstand corresponds to a level of about $30 \mathrm{KRad}$, and the $\mathrm{pH}$ parameter is not lower than 3.

Synrotrophic microbiological associations, including sets of different strains that are in a symbiotic state, are more effective. These microorganisms are not in the form of a simple mechanical mixture. They coexist in a synrotrophic association in such a state of joint symbiosis, when, in fact, they form a single macroorganism (although with separate systems of internal metabolism). In its scope, every member and every physiological group of the community is maximally adapted to the joint life activity and is in a state of collective mutual assistance and mutual protection. This system has a high degree of adaptation to various variations and "aggressive" manifestations of the external environment.

Obviously, such a system proves to be maximally adapted to changing aggressive conditions, which corresponds to their growth, including under the conditions of radiation. The effectiveness of this "collective defense" is extremely high. For example, in an acidic medium with $\mathrm{pH}=2$ (sufficiently concentrated hydrochloric acid) no "pure" strains can growth. At the same time, the synrotrophic association grows fine in such an environment after a certain transitional period. The same applies to large doses of radiation. Approximately, we can assume that the time interval of adaptation corresponds to a change of 5-10 generations, which allows us to estimate this interval from 10 hours to several days.

\section{EXPERIMENTS ON SYNTHESIS OF STABLE ISOTOPES IN MICROBIOLOGICAL SYSTEMS}

We began studying the nuclear processes in biological systems in the early 90s. The basis of the proposed experiments was the principle of "looking for what was lost under the street lamp" - which corresponds to the rule: if you have lost something in the dark, then you should look under the street lamp, because in other (dark) places you will not find anything! This principle gave priority to a reliable method of recording products of potential nuclear reactions, supplemented by the possibility of accumulating of these products. Based on our understanding of these processes, we investigated the reality of transmutation of stable isotopes based on the basic reaction ${ }^{55} \mathrm{Mn}+d={ }^{57} \mathrm{Fe}$ (see patent [9] and articles [10-11]), leading to the formation of a rare Mössbauer iron isotope ${ }^{57} \mathrm{Fe}$ in a medium based on heavy water, where along with the microorganisms and macronutrients necessary for the growth of microbial cultures, there was a manganese salt, but completely (or with a minimal admixture), there was no iron. The advantage of this reaction is that the final product can be identified by both "standard" and Mössbauer mass spectrometers. The nutrient composition is shown in Table 1.

Initial experiments were carried out on the basis of pure microbiological cultures of E. coli and Saccharomyces cerevisiae T-8. This reaction was successfully realized and identified with the help

Table 1.

Nutrient medium for transmuting manganese into iron.

\begin{tabular}{|l|c|c|c|}
\hline \multicolumn{1}{|c|}{ Components } & $\begin{array}{c}\text { Concentration } \\
\text { in medium (\%) }\end{array}$ & $\begin{array}{c}\text { Relative } \\
\text { admixture } \\
\text { of iron (no } \\
\text { more), } \%\end{array}$ & $\begin{array}{c}\text { Absolute } \\
\text { admixture of } \\
\text { iron (no more), } \\
\mathrm{g}\end{array}$ \\
\hline Sucrose & 3 & $10^{-4}$ & $3 \cdot 10^{-7}$ \\
\hline$\left(\mathrm{NH}_{4}\right)_{2}$ tartrate & 1 & $5 \cdot 10^{-4}$ & $5 \cdot 10^{-7}$ \\
\hline $\mathrm{MgSO}_{4} \cdot 7 \mathrm{H}_{2} \mathrm{O}$ & 0.25 & $2 \cdot 10^{-4}$ & $5 \cdot 10^{-8}$ \\
\hline $\mathrm{CaHPO}_{4} \cdot 7 \mathrm{H}_{2} \mathrm{O}$ & 0.008 & $1.5 \cdot 10^{-3}$ & $1.2 \cdot 10^{-8}$ \\
\hline $\mathrm{K}_{3} \mathrm{PO}_{4}$ & 0.5 & $5 \cdot 10^{-4}$ & $2.5 \cdot 10^{-7}$ \\
\hline $\mathrm{MnSO}_{4} \cdot 7 \mathrm{H}_{2} \mathrm{O}$ & 0.01 & $5 \cdot 10^{-4}$ & $5 \cdot 10^{-9}$ \\
\hline $\begin{array}{l}\mathrm{D}_{2} \mathrm{O}(\text { experiment) } \\
\mathrm{H}_{2} \mathrm{O} \text { (control) }\end{array}$ & $100 \cdot(10 \mathrm{ml})$ & $10^{-7}$ & $10^{-8}$ \\
\hline
\end{tabular}



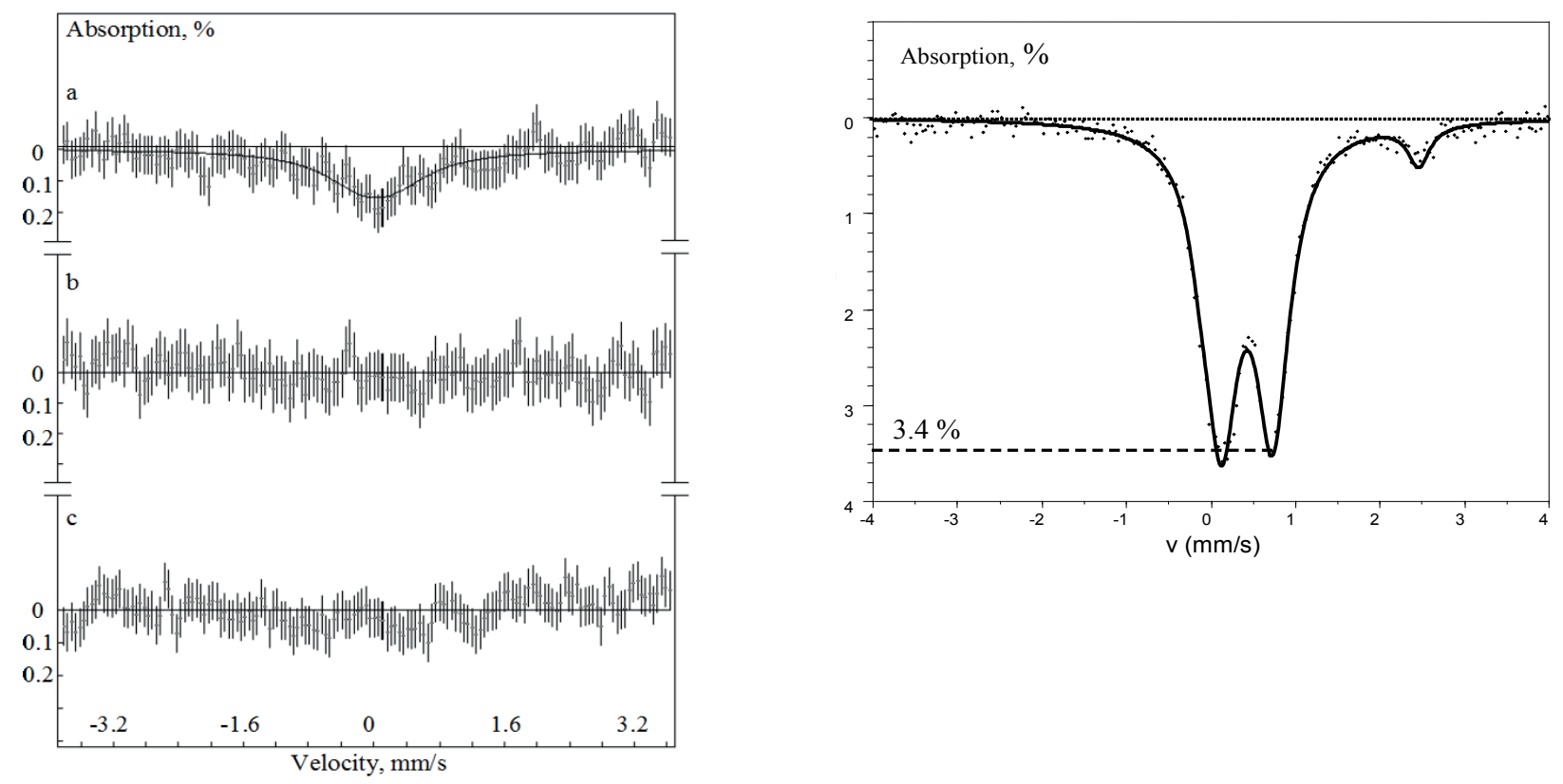

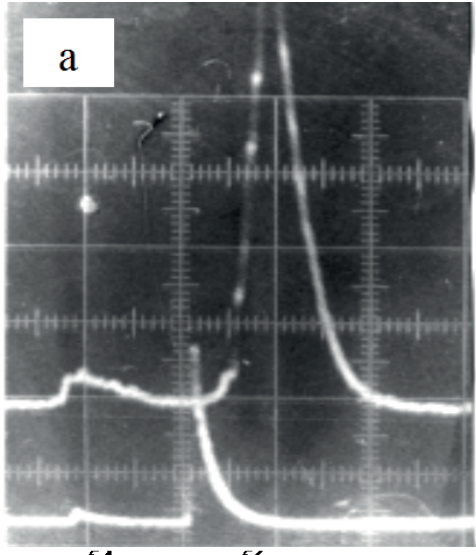

$\mathrm{Fe}^{54} \quad \mathrm{Fe}^{56}$

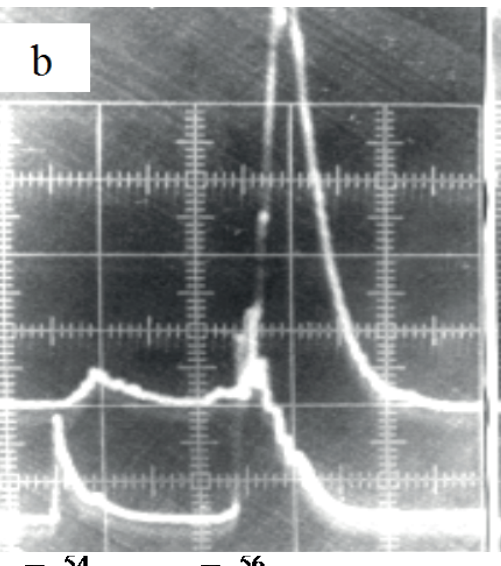

$\mathrm{Fe}^{54}$

$\mathrm{Fe}^{56}$

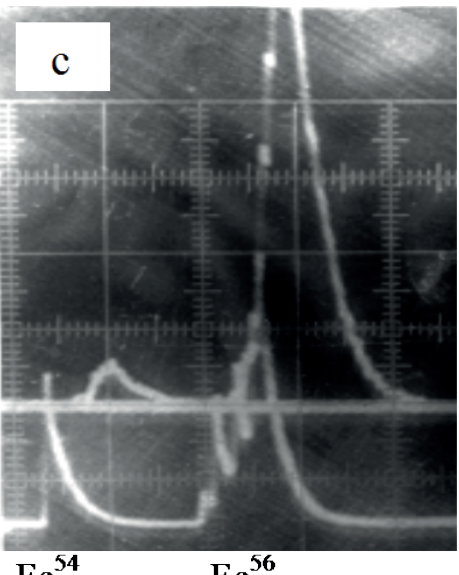

Fig. 1. The upper left is the Mössbauer spectrum of the dried microbiological culture of Saccharomyces cerevisiae T-8, grown in a liquid nutrient medium on the basis of: a) heavy water $\mathrm{D}_{2} \mathrm{O}$ in the presence of the ${ }^{55} \mathrm{Mn}$ isotope; b) light water $\mathrm{H}_{2} \mathrm{O}$ in the presence of ${ }^{55} \mathrm{Mn}$; c) heavy water $\mathrm{D}_{2} \mathrm{O}$ in the absence of the ${ }^{55} \mathrm{Mn}$ isotope. At the top right-Mössbauer spectrum of MCT granules grown in a nutrient medium on the basis of heavy water $\mathrm{D}_{2} \mathrm{O}$ in the presence of the ${ }^{55} \mathrm{Mn}$ isotope and the absence of iron (or its minimum amount as an impurity). Below - a fragment of the mass spectrum of the same culture, grown in a nutrient medium based on $\mathrm{H}_{2} \mathrm{O}$ in the presence of ${ }^{23} \mathrm{Na}$ and ${ }^{31} \mathrm{P}(\mathrm{b}),(\mathrm{c})$ and the absence of ${ }^{31} \mathrm{P}(\mathrm{a})$. The upper graphs on each of the photos below correspond to the control spectrum of the natural iron masses obtained in the same series as the corresponding lower graphs.

of Mössbauer and time-of-flight spectrometers (Fig. 1).

The typical duration of these experiments was $2 . . .3$ days, after which growth was stopped due to a change in acidity and self-intoxication of cultures by metabolic products.

The experimental efficiency of these reactions corresponded to the value $\lambda=\Delta N\left({ }^{57} \mathrm{Fe}\right) /$ $N\left({ }^{55} M n\right) \Delta t \approx 10^{-8}$ of the synthesized ${ }^{57} \mathrm{Fe}$ nuclei per one ${ }^{55} \mathrm{Mn}$ nucleus per second.

Further, another ${ }^{23} \mathrm{Na}+{ }^{31} \mathrm{P}={ }^{54} \mathrm{Fe}$ reaction was realized and investigated, with the formation of another rare iron isotope ${ }^{54} \mathrm{Fe}$. In transmutation experiments, $\mathrm{K}_{2} \mathrm{HPO}_{4}$ salt was present, and in control experiments it was absent. Corresponding results of mass-spectrometric analysis are presented in Fig. 1. The efficiency of this reaction was approximately the same as in the case of the synthesis of the ${ }^{57} \mathrm{Fe}$ isotope.

These experiments were further optimized by using microbial syntrophic associations of MCT ("microbial catalyst-transmutator"). The basis of MCT granules is the synthrophic association (superassociation) of many thousands of 
different types of microorganisms belonging to different physiological groups that represent different groups of microbial metabolism and are characterized by different mechanisms of microbial accumulation.

On the same Fig. 1, the Mössbauer spectrum of the dried MCT granules is presented. These granules grew for 30-50 days in a medium similar to the above discussed case of pure cultures. It is seen that the efficiency of transmutation in such a system is much higher.

Estimations made on the basis of analysis of these samples show that in the case of using of synrotrophic associations, the transmutation efficiency reaches a value $\lambda=\Delta N\left({ }^{57} \mathrm{Fe}\right) / N\left({ }^{55} \mathrm{Mn}\right) \Delta t$ $\approx 10^{-6}$ (synthesized ${ }^{57} \mathrm{Fe}$ nuclei per one ${ }^{55} \mathrm{Mn}$ nucleus per second).

Subsequent experiments were aimed at studying the possibility of transmutation not only of light and medium-weight isotopes, but also of heavy nuclei. The reaction of transmutation of a stable isotope of cesium into the barium isotope ${ }^{133} \mathrm{Cs}+p={ }^{134} \mathrm{Ba}$ was chosen as the object of research [12]. The expediency of such studies was due to the subsequent possibility of transmutation and deactivation of the ${ }^{137} \mathrm{Cs}$ radioactive isotope.

During the research, different types of synthrophic associations were used, adapted to aerobic or anaerobic conditions. The light-water nutrient medium contained glucose, dissolved base salts of the main macro- and microelements (including $\mathrm{NH}_{4} \mathrm{NO}_{3}, \mathrm{CaSO}_{4}, \mathrm{MgSO}_{4}$ ), as well as stable cesium as the $\mathrm{Cs}_{2} \mathrm{CO}_{3}$ salt, but did not contain the potassium vital for the growth of the crops included in these associations. Barium is a biochemical analogue of potassium and in its absence it can be used for the growth of microbiological cultures.

In the process of growth, samples were taken from the experimental cuvettes periodically, which were used to study the dynamics of the transmutation process. The results of these studies are shown in Fig. 2.

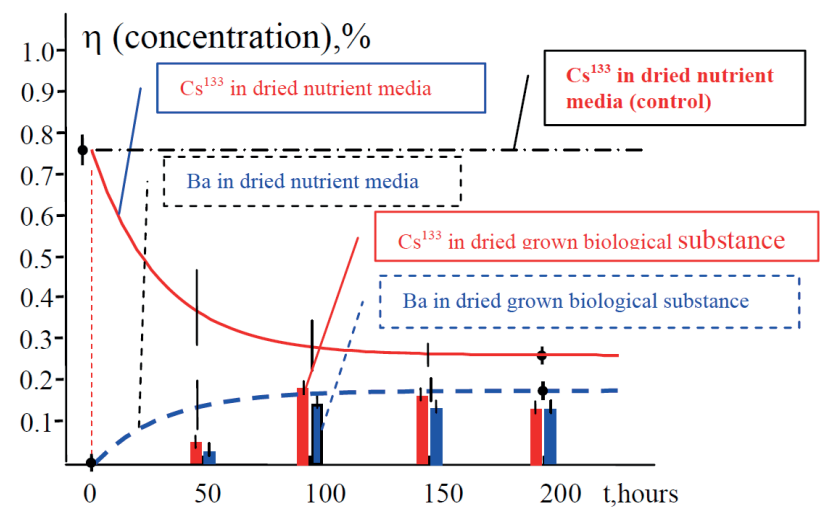

Fig. 2. The time dependence of the transmutation of a stable ${ }^{133} \mathrm{Cs}$ isotope into an ${ }^{134} \mathrm{Ba}$ isotope in a growing syntrophic association and in a nutrient medium.

From the obtained data it follows that the average efficiency of the transmutation reached the value $\lambda=N\left({ }^{134} B a\right) / N\left({ }^{133} C s\right) \Delta t \approx 10^{-6}$ synthesized nuclei of ${ }^{134} \mathrm{~B}$ a per core of ${ }^{133} \mathrm{Cs}$ per second.

The total amount of the ${ }^{133} \mathrm{Cs}$ isotope decreased from $\mathrm{M}_{133 \mathrm{Cs}} \approx 13 \mathrm{mg} /$ cell to $\mathrm{M}_{133 \mathrm{Cs}} \approx 6.5 \mathrm{mg} /$ cell for 192 hours.

\section{EXPERIMENTS ON TRANSMUTATION AND DETECTION OF RADIOACTIVE ISOTOPES IN MICROBIOLOGICAL SYSTEMS}

Currently, the task of safe storage, utilization and deactivation of radioactive waste from nuclear power plants is one of the main factors of the entire nuclear power industry. The urgency of this problem follows from simple digits.

The world currently has about 400 thousand tons of spent fuel elements (TVELs), more than 1 million tons of medium-level waste, the same amount of highly active water and more than 10 million tons of wastes of low activity. For example, in the territory of Hanford nuclear storage in the United States, there are 177 burial tanks (radwaste storage tanks) for liquid highlevel waste with a total volume of $204000 \mathrm{~m}^{3}$. According to estimates conducted in 2013, about $2 \%$ of these liquid wastes have already been released into the environment due to uncontrolled leaks from burial grounds.

Each year, these values increased by $5-7 \%$, and after the accident at the NPP in Fokushima, 
"reserves" of highly active water increase even faster.

By and large, the problem of utilization of radioactive waste has not yet been solved by anyone, and for spent fuel elements, there is no alternative for their storage for an indefinitely long period of storage after primary processing.

All the newest programs to create systems for utilization based on methods of "burning out" spent fuel with intensive neutron fluxes are extremely costly (each of several tens of billions of dollars) [13-17]. The analysis shows that a large number of new radionuclides will be formed in this case. This problem is well known in the problem of controlled thermonuclear fusion as a "first wall problem".

There is another direction of research related to the possibility of nuclear transformations under normal (not extreme) conditions in a substance, for example, at room temperature. In the 90 s of the 20th century this trend was called "cold nuclear fusion". At present, the term LENR ("nuclear reactions at low energy") is more used and closer to the essence of the processes. It is necessary to note the extremely ambiguous attitude of scientists towards such processes.

Most "classical" specialists in nuclear physics believe that the probability of nuclear transformations, if considered by the method of direct approximation of the physics of highenergy two-particle nuclear reactions to low energies, is very small, and such effects can not be a priori meaningful.

There is another point of view. It is actively developing recently and is based on a number of fundamental provisions of quantum mechanics. Its essence lies in the fact that direct approximation is not legal, since many nuclear physics processes at low energy are collective (not pairwise), are subject to the influence of the environment and can proceed with a fairly high probability, which makes them quite suitable for practical use. These results, in particular, directly follow from the specific behavior of the particle in a non-stationary potential well (in particular, in a nonstationary oscillator).

In recent years it has been shown [18-20] that one of the effective methods for a very significant increase in the transparency of a potential barrier at low particle energies is associated with the use of coherent correlated states (CCS) of particles interacting with the atoms (nuclei) forming this barrier. The most characteristic property of CCS is the possibility of forming controlled giant energy fluctuations of a particle whose amplitude can be thousands and millions times greater than the average (thermal) energy of a particle and reach values $\delta E \approx 10-50 \mathrm{keV}$. In a concentrated form, this is reflected in the modified uncertainty relations, called the Schrödinger-Robertson $\delta p \delta q \geq \hbar / 2 \sqrt{1-r_{p q}^{2}}, \quad \delta E \delta t \geq / 2 \sqrt{1-r_{E t}^{2}}$,

uncertainty relations, in which the product of the fluctuations of the corresponding dynamic variables (coordinate, momentum, energy, time, ...) is determined by the corresponding correlation coefficients, the magnitude of which is limited by the interval $0 \leq r_{p q}^{2} \leq 1, \quad 0 \leq r_{E t}^{2} \leq 1, \ldots$ [18-20].

In a stationary state in any system $r_{p q}^{2}, r_{E t}^{2} \ll 1$, these relations take the form of well-known Heisenberg uncertainty relations. Another situation in dynamic systems (including living objects). It is well known that the growth front of any biological object is never ideally homogeneous - local heterogeneities are always formed (in fact, these are potential nano-wells), which are leveled and eliminated during the growth process. Each of these nano-wells is a nonstationary oscillator for particles that are localized in it. In the process of dynamically changing of the parameters of these wells, CCS can be formed for these particles with a large value of the correlation coefficient $r_{p q}^{2}, r_{E t}^{2} \rightarrow 1$ and, accordingly, with unlimitedly increasing fluctuations of momentum and energy, which is enough to pass through the potential barrier [1820]. From this point of view, the growth zone of any biological object represents a collection of potential nanoreactors, in each of which a 
reaction involving these particles is possible, as well as the nuclei of atoms forming the "walls" of the dynamic nano-brain. Similar processes can occur in the space between cells during fission, in mitochondria, at the entrance to biological membranes, etc.

The correct interpretation and possible use of the experiments discussed below can change the view on the relationship between wildlife and the diversity of chemical elements and isotopes on Earth. The question concerns the possibility of nuclear transformations in biological systems under "non-reactor" conditions (room temperature, normal (atmospheric) pressure, absence of neutrons and charged particles of high energy). These issues, in particular, are considered and formalized in the patent [21].

Note that from the point of view of the possibility of catalytic effect on nuclear transmutations, the growing biological environment is no worse (and, most likely, better) than, for example, the usual crystal, which is most often used in LENR experiments. It is better in the sense that, unlike a static crystal, the growing biological system is a dynamic object, which allows us to consider each of its elements as a microscopic system of non-stationary potential wells and barriers.

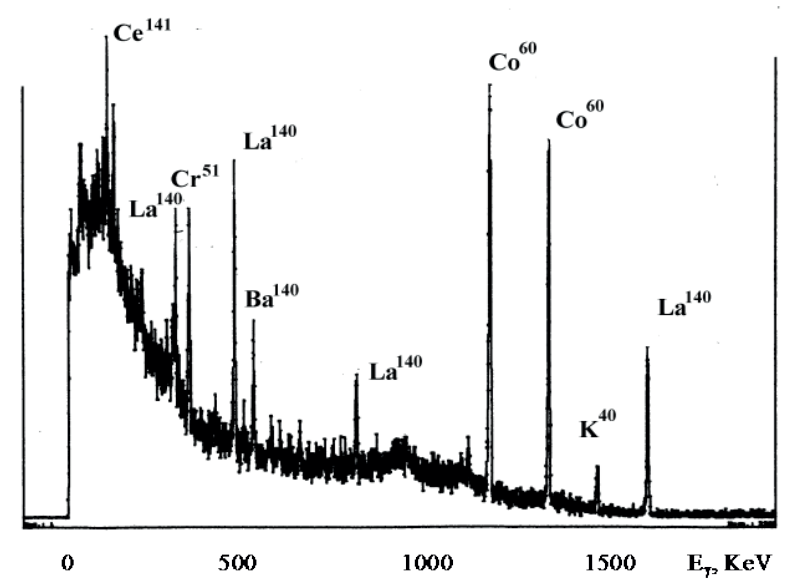

\subsection{Experiments on utilization of the reactor isotope ${ }^{140} \mathrm{Ba}$}

The realization of phenomena of heavy stable isotopes transmutation led to the formulation of the question about transmutation of radioactive nuclei. The first series of experiments was devoted to the possibility of influencing the activity of reactor water samples extracted from the first circuit of the water-water nuclear reactor, which is located in the Institute of Nuclear Research in Kiev.

The water under study had an activity of about $10^{-4}$ Curie/L and contained a number of highly active unstable isotopes (in particular, ${ }^{24} \mathrm{Na},{ }^{40} \mathrm{~K},{ }^{60} \mathrm{Co},{ }^{91} \mathrm{Sr},{ }^{131} \mathrm{I},{ }^{135} \mathrm{Xe},{ }^{140} \mathrm{Ba},{ }^{140} \mathrm{La},{ }^{141} \mathrm{Ce}$, ${ }^{239} \mathrm{~Np}$ ). The gamma-radiation spectrum of the reactor isotopes contained in this water is shown in Fig. 3. Water samples of the same volume (about $5 \mathrm{ml}$ ) were placed in the same glass thinwalled closed cuvette with a volume of about $10 \mathrm{ml}$. In a part of the cuvettes with active water, the same mass of MCT granules was placed.

The remaining cuvettes with water (but without granules) were controls. The essence of the research consisted in a periodic (after 5 days) study of the gamma spectrum of active water. To eliminate the influence of the distance factor (it may be associated with a small increase

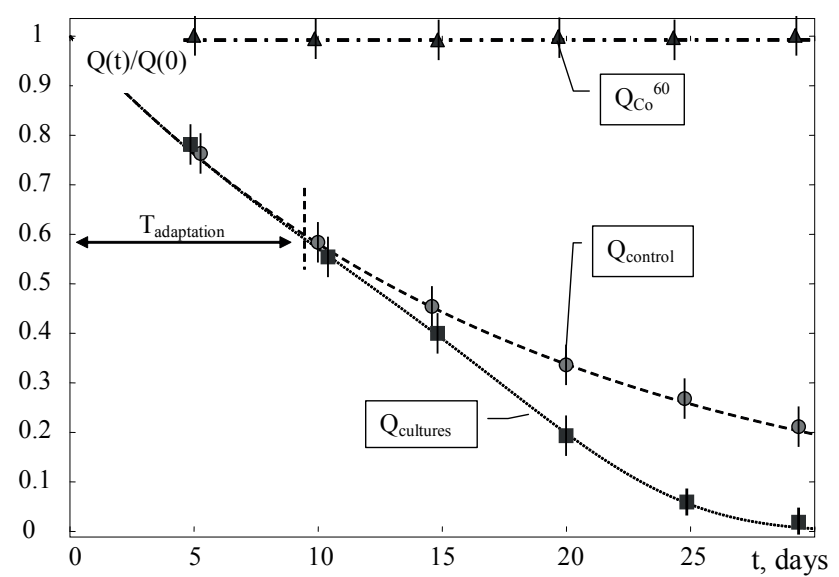

Fig. 3. Left: spectrum of gamma radiation of distilled water from the first circuit of the water-water nuclear reactor. The data correspond to the tenth day after the sampling of water from the core of the reactor. On the right is the dependence of the $Q(t)$ activity of ${ }^{140} \mathrm{Ba},{ }^{140} \mathrm{La}$ and ${ }^{60} \mathrm{Co}$ reactor isotopes in reactor water samples in the transmutation experiment (here $Q_{\text {cultures }}$ is activity of cuvettes at the presence of metabolically active microorganisms) and in control cuvettes without microorganisms $\left(Q_{\text {control }}\right.$ activity): $Q_{\text {control }}-$ decay of ${ }^{140} \mathrm{Ba}$ and ${ }^{140} \mathrm{La}$ in control experiments without MCT granules, $T_{\text {adatation }}$ - time of internal adaptation of microbial synthrophic association to action of radiation, $Q_{\text {cultures }}$ - deactivation of ${ }^{140} \mathrm{Ba}$ and ${ }^{140} \mathrm{La}$ isotopes in transmutation experiments with presence of $M C T$ granules, $Q_{\text {Co-60 }}-$ activity of ${ }^{60} \mathrm{Co}$ isotope in control and experimental cuvettes with MCT. 
in the volume of a mixture of water and granules with the growth of microbiological cultures or their spatial redistribution), we used an amplitude germanium detector with a large size of germanium crystal, in which the cells under study were arranged in turn exactly in the center.

Fig. 3 shows the averaged results of the dependence of the activity of the isotopes ${ }^{140} \mathrm{La}$ and ${ }^{60} \mathrm{Co}$ in cuvettes with MCT granules $\left(Q_{\text {cultures }}\right)$ and in control cells $\left(Q_{\text {control }}\right)$ from the time after the beginning of the experiments.

From the data obtained it follows that the activity of the long-lived ${ }^{60} \mathrm{Co}$ isotope remains constant throughout the measurement period (about 40 days) (both in the control cuvettes and in cuvettes with MCT granules). This indicates that systematic methodological errors (a change in the volume of the liquid, a change in the position of the cell relative to the center of the crystal of the detector, etc.) did not have a significant effect on the result.

At the same time, a fundamentally different law for the reduction of the activity of the isotope ${ }^{140} \mathrm{La}$ in both types of cuvettes was discovered. This isotope has a relatively short lifetime $\left(\tau_{\mathrm{La}}=40.3\right.$ hours $)$ and is a daughter unstable isotope of the longer-lived ${ }^{140} \mathrm{Ba}$ isotope, whose lifetime is $\tau_{\mathrm{Ba}}=12.7$ days.

The initial activities of the ${ }^{140} \mathrm{Ba}$ and ${ }^{140} \mathrm{La}$ isotopes (on the 10th day after extraction the water from the reactor core) for each of the cuvettes was, respectively, $Q_{\mathrm{Ba}-140}=1.46 \cdot 10^{-7}$ Curie/L and $Q_{\mathrm{La}-140}=2.31 \cdot 10^{-7}$ Curie/L. Since $\tau_{\mathrm{La}}<<\tau_{\mathrm{B}}$, the observed decrease in ${ }^{140} \mathrm{La}$ activity reflected a decrease in activity of ${ }^{140} \mathrm{Ba}$.

It was found that the decrease in activity of ${ }^{140} \mathrm{La}$ in control cuvettes approximately corresponded to the law of "standard" decay of the ${ }^{140} \mathrm{Ba}$ isotope with a "tabulated" lifetime. Approximately the same law of decreasing activity of ${ }^{140} \mathrm{La}$ was observed in cuvettes with granules up to 10 days of the experiment. However, after this, periodic measurements showed that the rate of decrease in activity of
${ }^{140} \mathrm{La}$ (and hence of activity ${ }^{140} \mathrm{Ba}$ ) is equivalent to a more accelerated decay. Extrapolation shows that the effective lifetime of this isotope is reduced by about 2 times in relation to the lifetime of the ${ }^{140} \mathrm{Ba}$ isotope.

These results can be explained on the basis of the assumption that the ${ }^{140} \mathrm{Ba}$ isotope could be converted in a cuvette with MCT granules to another, non-radioactive isotope. In this case, the presence of an initial, unchanged region in the law of decay can be explained by the processes of adaptation of the microbiological association to the action of radioactive irradiation in a cell with active water. This time (about 10 days) correlates well with the expected time of the change of 10 generations of microbiological cultures.

Analysis of possible isotope transformations has shown that in this case the following transmutation reaction of the radioactive ${ }^{140} \mathrm{Ba}$ isotope to a stable nucleus of another type ${ }^{140} \mathrm{Ba}+{ }^{12} \mathrm{C}={ }^{152} \mathrm{Sm}+\Delta \mathrm{E}$ is possible. This reaction is energy-efficient and is characterized by a positive reaction energy

$\Delta \mathrm{E}=\mathrm{E}\left(A_{\mathrm{Ba}} Z_{\mathrm{B} a}\right)+\mathrm{E}\left(A_{\mathrm{C}}, Z_{\mathrm{C}}\right)-\mathrm{E}\left(A_{\mathrm{Sm}}, Z_{\mathrm{Sm}}\right)=8.5 \mathrm{MeV}$.

The carbon required for this reaction is abundant in the volume of MCT granules.

There is one more question about the biochemical aspects of the expediency of the course of this particular reaction in the living system. For answer to this question, it should be noted that the law of constancy of the chemical composition of biological objects is one of the fundamental properties of living matter. If we proceed from this circumstance, then it is obvious that according to the analysis of biochemical aspects of nuclear processes [11, 12], the isotope transmutation reaction in the biological system will be possible if the result of the reaction is an isotope corresponding to such a chemical element that either itself is included in the number of vital chemical elements (such as $\mathrm{H}, \mathrm{O}, \mathrm{C}, \mathrm{K}, \mathrm{Ca}, \mathrm{N}, \mathrm{P}, \mathrm{Fe}, \ldots$ ), or it is a biochemical analogue of such an element. In the latter case, it should have approximately the same ionic radius and, preferably, the same valence. In this case, 
the reaction efficiency will be great only when the vital chemical element or its biochemical analogue is not contained in the nutrient medium or is contained in a small amount.

A simple comparison shows that $\mathrm{Sm}^{2+}$ and $\mathrm{Ca}^{2+}$ ions are biochemical analogues and have a close ionic radius in the divalent state $\left(\mathrm{R}_{\mathrm{Sm}} \approx 1.2 \AA, \mathrm{R}_{\mathrm{Ca}} \approx 1.06 \AA\right)$. As you know, calcium is among the vital elements, and its concentration in the volume of granules was small. In this case, we can assume that the growing microbiological association could fill the lack of calcium with a synthesis of its biochemical analog (samarium). At the same time, the unchanged activity and the number of nuclei of the ${ }^{60} \mathrm{Co}$ isotope can be explained by the fact that in this system there is no possible energy-efficient transmutation reaction in which ${ }^{60} \mathrm{Co}$ participates and whose product would be an isotope of that vital chemical element that is absent in the nutrient medium. In other words, ${ }^{60} \mathrm{Co}$ may simply be "not in demand".

\subsection{Experiments on utilization of the} long-lived reactor ${ }^{137} \mathrm{Cs}$ isotope in growing associations of microbiological structures

Nontrivial results, indicating an abnormal decrease in the activity of ${ }^{140} \mathrm{Ba}$ and ${ }^{140} \mathrm{La}$ isotopes in experiments with reactor water in the presence of microbiological cultures, stimulated the setting up of special experiments to study the possibility of utilization of long-lived "fuel" isotopes formed during the operation of nuclear reactors. These works were conducted with the participation of our colleagues V.N. Pavlovich and A. Odintsov from the Institute for Nuclear Research and the Institute for Nuclear Safety Problems in Kiev [22]. The microbiological granules of MCT were prepared by our colleague A.B. Tashirev from the Institute of Microbiology in Kiev.

The utilization of such isotopes is of the greatest practical interest. Consider these experiments in more detail. In these experiments we have used the same closed glass cuvettes, each containing $10 \mathrm{ml}$ of distilled water, in which the salt containing ${ }^{137} \mathrm{Cs}$ was dissolved. The total activity of each of the cuvettes was about $2 \cdot 10^{4} \mathrm{bq}$.

The scheme of investigations is shown in Fig. 4. The same mass of MCT granules was placed in 7 cuvettes. In 6 cuvettes, purified salts of $\mathrm{K}, \mathrm{Ca}, \mathrm{Na}, \mathrm{Fe}, \mathrm{Mg}$ and $\mathrm{P}$ were added to the active water. These chemical elements are among the most vital for any living system. The main purpose of using of such additives was to find ways to block possible channels of transmutation because if a specific chemical element is present in the system from the number of vital ones, then the assimilation of its biochemical analogue during transmutation becomes unlikely. In addition, such substitutions were carried out with the goal of creating the optimal composition of microelements for rapid growth of microorganisms. The results obtained below confirm the importance of such substitutions. Two additional cuvettes were used

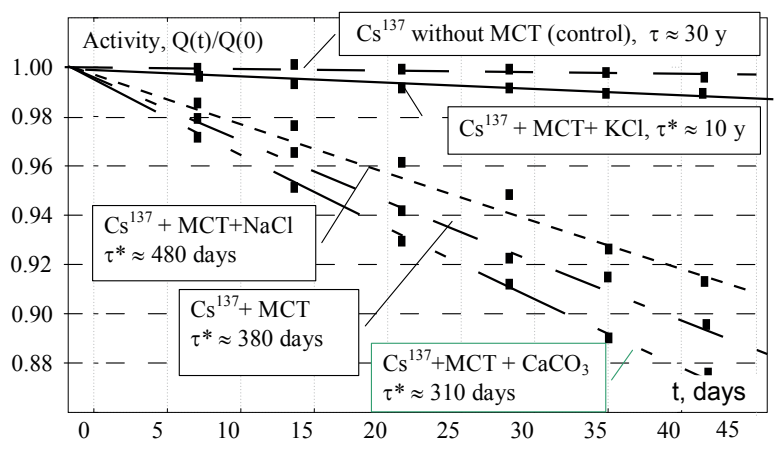

Fig. 4. Scheme of studies of the utilization of active isotopes under different conditions and the results of experiments on the accelerated utilization (deactivation) of the ${ }^{137} \mathrm{Cs}$ isotope in "biological cells" in the presence of MCT and various chemical elements. 
for monitoring: one contained active water and MCT (but did not contain additional salts), and the other - only active water.

All the cuvettes were closed and kept at a temperature of $20^{\circ} \mathrm{C}$. The amplitude gammaray spectrum of the cuvette was measured every 7 days on the same detector, in which a Ge crystal was used. Particular attention was paid to reducing the influence of errors associated with the measurement process. For this purpose, we have used cuvettes with a low height, and the detector with a large Ge crystal. The cuvettes were set at the same position in the center of the crystal of the detector for each measurement.

The results of the change in the relative activity of the ${ }^{137} \mathrm{Cs}$ isotope are shown in Fig. 4 and in Table 2.

From the obtained data it follows that during a 100-day observation period, a steady decrease in ${ }^{137} \mathrm{Cs}$ isotope activity was recorded in all cuvettes containing active water and MCT. In the control cuvette containing only active water, the change in the activity of ${ }^{137} \mathrm{Cs}$ isotope corresponded to the standard spontaneous decay with a lifetime of about 30 years. For other investigated isotopes, the changes were much weaker.

The fastest decrease in activity (it was equivalent to a decrease in the lifetime of ${ }^{137} \mathrm{Cs}$ by a factor of 35 to $\tau^{*} \approx 310$ days) was observed in a cuvette containing a calcium salt. This decrease in activity was not related to the accelerated decay, but was the result of reaction of transmutation the radioactive ${ }^{137} \mathrm{C}$ s isotope to the stable isotope of another element.

Table 2.

Changes in the activity of ${ }^{137} \mathrm{Cs}$ in the "optimal" medium (MCT + active water $+\mathrm{CaCO}_{3}$ salt)

\begin{tabular}{|l|l|l|c|c|c|}
\hline Izotope & $\begin{array}{c}\text { Energy, } \\
\mathrm{keV}\end{array}$ & $\begin{array}{c}\text { The beginning } \\
\text { of the } \\
\text { experiment. } \\
N_{1}-\text { the } \\
\text { number of } \\
\text { events } 10^{3} \text { sec. }\end{array}$ & $\begin{array}{c}\text { The end } \\
\text { of the } \\
\text { experiment } \\
(100 \text { days). } \\
N_{2}-\text { the } \\
\text { number of } \\
\text { events } 10^{3} \\
\text { sec. }\end{array}$ & $\begin{array}{c}\text { Spon- } \\
\text { taneous } \\
\text { decay }\end{array}$ & $\begin{array}{c}\text { Change } \\
\text { of } \\
\text { activity }\end{array}$ \\
\hline${ }^{137} \mathrm{Cs}$ & 661.7 & 266900 & 216800 & $-0.6 \%$ & $-24 \%$ \\
\hline
\end{tabular}

An analysis of possible ways of transmutation should be based on several fundamental logical premises:

- this process should be energy-efficient, i.e. transmutation should be characterized by a positive reaction energy $\Delta E \geq 0$;

- the transmutation reaction must in a certain sense be adapted to the biological system, the result of the reaction must be an isotope corresponding to one of the vital elements or its biochemical analogue;

- the initial isotope of the transmutation reaction should reflect (correlate) the quantitative composition of the medium;

- among the different initial isotopes that can participate in reaction, preference should be given to the lightest isotopes for which the effect of potential barriers should be the smallest among the possible ones.

Based on these conditions, we can expect that the utilization of ${ }^{137} \mathrm{Cs}$ is related to the reaction ${ }^{137} \mathrm{Cs}+{ }^{1} \mathrm{p}={ }^{138} \mathrm{Ba}+\Delta E$, flowing with the participation of water protons. The result of the reaction is the stable isotope ${ }^{138} \mathrm{Ba}$. The reaction energy is positive and equal to $\Delta E=5.58 \mathrm{MeV}$.

Concerning the "biological expediency" of such a hypothesis, it can be noted that $\mathrm{Ba}^{2+}$ and $\mathrm{K}^{+}$ions are biochemical analogs: they have approximately the same ionic radii in the divalent state $\left(R_{\mathrm{Ba}} \approx 1.4 \AA, \mathrm{R}_{\mathrm{K}} \approx 1.33 \AA\right)$. Since the replaceable element (potassium) is one of the vitally important trace elements, the probability of such a substitution is quite large and the ions of the synthesized barium can replace potassium ions in metabolic processes with the growth of cultures. Such

a substitution appears to be more effective than the "direct" replacement of potassium by cesium in the case of potassium deficiency (this can be seen from the large difference in the ionic radii of cesium $R_{\mathrm{Cs}} \approx 1.65 \ldots 1.69 \AA$ and potassium $R_{\mathrm{K}} \approx 1.33 \AA$ ). It should be noted that a similar replacement of ions was previously observed and analyzed in experiments with 
the microbiological culture of Blastocladiella emersonii [23]. In these experiments, a fairly effective replacement of $\mathrm{K}^{+}$ions by $\mathrm{Rb}^{+}$ and $\mathrm{Ba}^{2+}$ ions was recorded. These ions can replace each other in the processes associated with ion transport through the membrane into the cell.

Another interesting question relates to the cause of increased efficiency of utilization when using an additional calcium salt. Apparently, this effect is associated with the general pattern of the metabolism of microbiological cultures: the optimal growth of culture corresponds to the necessary balance of all micro- and macroelements. It is possible that it was calcium deficiency that was the "bottleneck" that inhibited the growth process and accompanying transmutation in a particular growing microbiological system.

In recent years, further improvements in bio- and nuclear technology has led to significant progress of these processes using more optimal biological substances and their modes of growth. Recent experiments have shown that the recycling process can be accelerated many times. In particular, the mean (according to parallel experiments) decrease in ${ }^{137} \mathrm{Cs}$ concentration over 14 days was $23 \%$, which corresponds to an acceleration of deactivation more than 200 times. In some cases, a decrease in the concentration of ${ }^{137} \mathrm{Cs}$

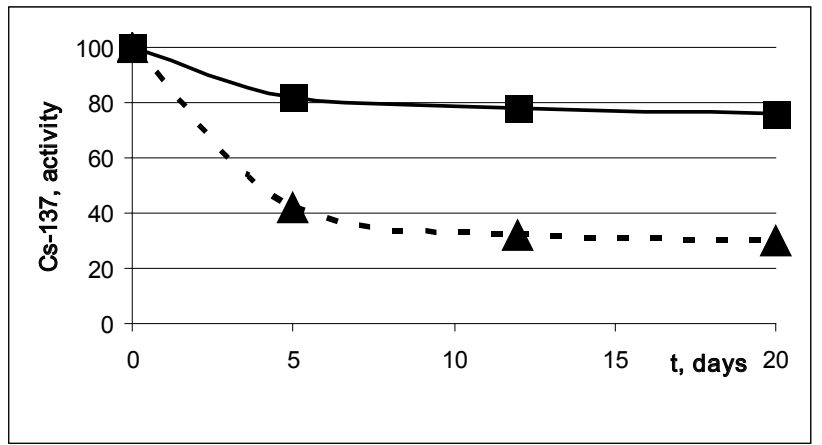

Fig. 5. Reduction of the activity of the aqueous solution of ${ }^{137} \mathrm{Cs}$ in the optimized syntrophic association (the upper graph is the average data for the series of experiments, the lower is the most optimal case). in these experiments reached $40 \%$ and even $70 \%$ (Fig. 5) [12].

\section{CONCLUSION}

The obtained results testify to the reality of the process of transmutation of both stable and radioactive isotopes accompanying the growth of microbiological cultures. The authors are aware that the totality of the observed phenomena and, especially, their interpretation do not coincide with the traditional notions and can cause serious criticisms. On the other hand, such processes have a purely physical basis and are not directly related to the specific features of the growth of living organisms, although they are stimulated by such a process. The authors have several patents (including international ones) on this modern technology.

In our minds, certain stereotypes are firmly fixed, among which the question of the "industrial" character of nuclear transformations is one of the most stable. Based on previous experience in the creation and operation of large-scale nuclear power, it is difficult to imagine that such processes (but in incomparably smaller scales) can take place in a living organism. However, by and large, there is no significant opposition. The whole question is only in the appropriateness of such transformations and in their very low efficiency caused by mutual repulsion of the nuclei due to the presence of the Coulomb barrier. At the molecular level, the specificity of the interaction and motion of microparticles is fully described by the laws of quantum mechanics and electrodynamics for both living and nonliving objects. From this point of view, there is no difference between them! As the analysis [18-20] shows, in nonstationary systems of the potential well type, an interference shortterm suppression of such repulsion is possible due to the formation of large fluctuations of momentum and kinetic energy in the process of formation of coherent correlated states. Such processes can be successfully implemented in 
any system if the necessary prerequisites are met.

It is obvious that such nuclear reactions can not be called a semi-mystical term "biological transmutation". These processes are ordinary nuclear reactions, but they are realized in growing biological systems and under the catalytic effect of dynamic electric fields accompanying atomic-molecular processes that take place with the growth of these systems. In any case, the experiment plays a decisive role. Such a process can be called "nonstationary dimensional nuclear catalysis."

In our understanding, the influence of the biological object itself on the process of nuclear transformation is in the following circumstances:

- the dynamic spatial structure of the growing bioobject at the molecular and atomic levels acts as a "spatial catalyst" for the nuclear reaction, which ensures a short-term elimination of the Coulomb barrier factor of the reaction;

- specificity of the growth of a particular biological object provides selective functions that characterize the effectiveness of a particular reaction.

In conclusion, we can say a few words about the biological specifics of the observed phenomena. The very phenomenon of the transformation of chemical elements and isotopes in biological systems is based, first of all, on the repeatedly verified condition of constancy of the elemental composition of microorganisms. From this fact follows the heuristic assumption that in the case when some of the required macro- or microelements are missing in the system, there are prerequisites that they will be synthesized and fixed in a finite form as a result of physically grounded nuclear reactions.

In conclusion, we note that the words of V.I. Vernadsky that the biosphere is a natureforming and relief-forming factor in the evolution of the Earth can be supplemented by the observation that it can be an isotopetransforming factor that significantly changes the distribution and quantity of different elements and isotopes.

\section{REFERENCES}

1. Biberian JP. Biological transmutations. Current science, 2015, 108(4):633.

2. Biberian JP. Biological transmutations: historical perspective. Journal Condens. Matter Nucl. Sci., 2012, 7:11.

3. Kervran CL. Transmutations Biologiques, Métabolismes Aberrants de l'Azote, le Potassium et le Magnésium. Librairie Maloine S.A., Paris, 1963.

4. Kervran CL. A la Découverte des Transmutations Biologiques. Librairie Maloine S.A., Paris, 1966.

5. Kervran CL. Preuves Relatives à l'Existence de Transmutations Biologiques. Librairie Maloine S.A., Paris, 1968.

6. Kervran CL. Biological Transmutations. Happiness Press, USA, Magalia, California, 1998.

7. Vysotskii VI, Kornilova AA. Nuclear fusion and transmutation of isotopes in biological systems. Moscow, Mir Publ., 2003.

8. Vysotskii VI, Kornilova AA. Nuclear transmutation of stable and radioactive isotopes in biological systems. India, Pentagon Press, 2010.

9. Vysotskii VI, Kornilova AA. Sposob polucheniya stabilnykh izotopov za schet yadernoy transmutatsii tipa nizkotemperaturnogo yadernogo sinteza elementov v mikrobiologicheskikh kulturakh [A method of producing stable isotopes due to nuclear transmutation type lowtemperature nuclear fusion of elements in microbiological cultures]. Patent $R U$ 2052223C1, 1995 (https://patents.google. com/patent/RU2052223C1/ru) (in Russ.).

10. Vysotskii VI, Kornilova AA. Transmutation of stable isotopes and deactivation of radioactive waste in growing biological systems. Annals of Nuclear Energy, 2013, 62:626.

11. Vysotskii VI, Kornilova AA. Microbial Transmutation of Cs-137 and LENR in 
growing biological systems. Current Science, 2015, 108:142.

12. Vysotskii VI, Kornilova AA, Gaidamaka S, Kashcheev V, Zhura E, Tserbaev A. Biotransmutation of Cs133 and Biodeactivation of Cs137 by Aerobic Microorganisms of Methanogenic Sea Ooze. 20th Int. Conf. on Condensed Matter Nuclear Science (ICCF-20), 2-7 Oct 2016, Japan, Sendai. Book of Abstracts, p. A91.

13. Minato K, Ikegami T, Inoue T. Recent research and development activities on partitioning and transmutation of radioactive nuclides in Japan. Actinide and Fission Product Partitioning and Transmutation. Eight Information Exchange Meeting. USA, Las-Vegas, November 2004, p.29-47.

14. Savage C. Overview of United States P\&T Programme. Ibid, p. 49-56.

15. Ponomarev LI. Overview of Russia P\&T Programme. Ibid, p. 79-90.

16. Stanculescu A. IAEA Activities in the aria of partitioning and transmutation. Ibid, p.101-108.

17. Radioactive Waste Management. World Nuclear Association. July 2015. Retrieved 2015-08-25.

18. Vysotskii VI, Adamenko SV, Vysotskyy MV. Acceleration of low energy nuclear reactions by formation of correlated states of interacting particles in dynamical systems. Annals of Nuclear energy, 2013, 62:618.

19. Vysotskii VI, Vysotskyy MV. Coherent correlated states and low-energy nuclear reactions in non stationary systems. European Phys. Journal, 2013, A49:99.

20. Vysotskii VI, Vysotskyy MV. Coherent correlated states of interacting particles the possible key to paradoxes and features of LENR. Current Science, 2015, 108:524.

21. Kornilova AA, Vysotskii VI. Method for purifying water of radionuclides. Intern. patent WO 2015156698 A1, 11 April 2014.

22. Vysotskii VI, Odintsov A, Pavlovich VN, Tashirev AB, Kornilova AA. Experiments on controlled decontamination of water mixture of long-lived active isotopes in biological cells. Proc. 11th Int. Conf. on Condensed Matter Nuclear Science, 2004, France, Marseilles, World Scientific, Singapore, 2006, p. 530-536.

23. Van Brunt J, Caldwell JH, Harold FM. Circulation of potassium across the plasma embrane of Blastocladiella emersonii: K-chanel. J. Bacteriol., 1982, 150(3):1449. 